in this study. Eligible patients must have received a script for a reimbursed DMT for RRMS between September 2011 and February 2016. Patients were classified into five age-groups (ages $18-30 ; 31-40 ; 41-50 ; 51-60 ; 61+$ ) and defined as persistent if their DMT script was filled within 4 months. Persistence was derived using the Kaplan-Meier method and hazard ratios (HR) to represent the relative rate of drop-off of different age groups.

Results Patients aged 18-30 $(\mathrm{n}=250)$ had a 44\% increased risk of discontinuation (HR 1.44 (95\%CI: 1.22-1.72) compared to the 'all ages' cohort $(n=1,866)$; no significant difference was observed for any other age group (HRs between 1.08 and 0.92). Patients in this 18-30 age-group had a significantly higher risk of discontinuation on injectable therapy (glatiramer acetate, interferon beta- $1 \mathrm{a}$, interferon beta- $1 \mathrm{~b}$ ) compared to those on non-injectable therapy (dimethyl fumarate, fingolimod, natalizumab, teriflunomide) (HR $2.42 \quad(95 \%$ CI: $1.63-3.61)$.

Conclusions Patients aged 18-30 were the least persistent age group in this study. Patients aged 18-30 were less persistent on injectable than non-injectable DMTs.

\section{OVERLAPPING SYNDROME OF GIANT CELL ARTERITIS AND ANCA-ASSOCIATED VASCULITIS COMPLICATED BY SEVERE AXONAL NEUROPATHY: A CASE REPORT}

'Laura Perju-Dumbrava*, ${ }^{1}$ Fariha Islam, ${ }^{1}$ Rohitha Makonahalli, ${ }^{2}$ Catriona McLean, ${ }^{3}$ Hedley Griffiths, ${ }^{1}$ Abhishek Malhotra. ${ }^{1}$ Neurology, Barwon Health, Geelong, VIC, Australia; ${ }^{2}$ Anatomical Pathology, Alfred Hospital, Melbourne, VIC, Australia; ${ }^{3}$ Rheumatology, Barwon Health, Geelong, VIC, Australia

\subsection{6/jnnp-2019-anzan.45}

Case A 61-year- old woman presented with what was thought to be a refractory, temporal artery biopsy-proven giant cell arteritis (GCA). After 4 months of therapy she was unable to get the prednisolone dose below $40 \mathrm{mg} /$ day, so weekly subcutaneous Tocilizumab was introduced but with minimal response after 8 weeks. She was then admitted to hospital with a severe rapidly progressive length dependent sensorimotor peripheral neuropathy. Nerve conduction studies showed predominantly an axonal neuropathy with multiple pseudoconduction blocks.

Sural nerve and gastrocnemius biopsies revealed a necrotising vasculitis with numerous giant cells and active axonal degeneration. She proved to be ANCA-PR3 positive, without other systemic manifestations. ANCA-associated vasculitic neuropathy was diagnosed and she was treated with two 1g Rituximab infusions, 2 weeks apart.

On follow-up after a month, she had regained some strength but still required a wheelchair for mobility. Further Rituximab infusion after 6 months is planned; prednisolone had been successfully weaned to $10 \mathrm{mg} /$ day.

Conclusion Peripheral nerve involvement, which is relatively common in ANCA-associated vasculitis, has also been reported in $14 \%$ of GCA cases. But ANCA-PR3 positivity is rare in biopsyproven GGA, with only a handful of well-documented cases.

Heightened suspicion of an alternative diagnosis in the face of an unusual clinical course (lack of steroid response and appearance of small vessel vasculitic symptoms for what is accepted to be a large vessel vasculitis) is critical and our experience highlights the important fact that a diagnosis of one of these disorders does not preclude the subsequent diagnosis of the other.

\section{GLUT-1 DEFICIENCY PRESENTING AS DOPA-RESPONSIVE DYSTONIA: AN ATYPICAL PHENOTYPE OF A RARE DISEASE}

${ }^{1}$ Sophie E Waller*, 'Jeffrey Blackie, ${ }^{2}$ Himanshu Goel. ${ }^{1}$ Department of Neurology, John Hunter Hospital, New Lambton Heights, NSW, Australia; ${ }^{2}$ John Hunter Hospital, New Lambton Heights, NSW, Australia

\subsection{6/jnnp-2019-anzan.46}

Introduction The SLC2A1 gene encodes the glucose transporter GLUT1, responsible for normal glucose transport across the blood-brain barrier. Mutations in this gene have classically been associated with an epileptic encephalopathy referred to as GLUT-1 Deficiency Syndrome, which typically presents with early-onset refractory epilepsy, developmental delay and complex movement disorders. ${ }^{1} 2$ More recently, SLC2A1 variants have been identified in patients with paroxysmal exerciseinduced dyskinesia (PED), with or without a history of epilepsy. ${ }^{3} 4$ Response to medication is typically poor; however, both seizures and dyskinesia may improve following implementation of a ketogenic diet. ${ }^{5}$ A single case of levodopa responsiveness has previously been described in a subject with SLC2A1 mutation and PED. ${ }^{5}$

Methods and results We describe a 47-year-old female with mild intellectual disability since childhood but no history of epilepsy, who developed episodic dystonia affecting the lower limbs in her early 20's. A clinical diagnosis of dopa-responsive dystonia was made following a marked, sustained response to levodopa. There was no significant family history. In her 40's she developed breakthrough dystonia with exertion and choreiform movements affecting the fingers and face. A subsequent dystonia panel identified a heterozygous variant c.[1199G $>$ T]; [=] (p.Arg400Leu) in the SLC2A1 gene. Cerebrospinal fluid glucose concentration was low $(2.0 \mathrm{mmol} / \mathrm{L})$. She declined a trial of a ketogenic diet.

SLC2A1 variants are associated with PED; however, response to levodopa has not been widely reported.

Conclusion It is becoming increasingly evident that the phenotypic presentations of GLUT1 deficiency are diverse, and SLC2A1 testing should be considered in a broader range of patients.

\section{REFERENCES}

1. Wang, D, Kranz-Eble $P$, De Vivo DK. Mutational analysis of GLUT1 (SLC2A1) in Glut-1 Deficiency Syndrome. Human Mutation 2001;16:224-231.

2. Klepper J, Leiendecker B. GLUT1 deficiency syndrome - 2007 update. Developmental Medicine \& Child Neurology 2007;49:707-716.

3. Schneider SA, Paisan-Ruiz C, Garcia-Gorostiaga I, Quinn NP, Weber YG, et al. GLUT1 gene mutations cause sporadic paroxysmal exercise-induced dyskinesias. Mov Disord 2009;24(11):1684-1696.

4. Suls A, Dedeken P, Goffin K, Van Esch H, Dupont P. Paroxysmal exercise-induced dyskinesia and epilepsy is due to mutations in SLC2A1, endoding the glucose transporter GLUT1. Brain 2008;131:1831-1844.

5. Baschieri F, Batla A, Erro R, Ganos C, Cordivari C, Bhatia KP. Paroxysmal exercise-induced dystonia due to GLUT-1 mutation can be responsive to levodopa: a case report. J Neurol 2014;261:615-616.

\section{IMPACT OF ONLINE ADULT HEADACHE GUIDELINE ON HEADACHE REFERRAL TO NEUROLOGY CLINIC}

Leon Huang, David Bourke*, Anna Ranta. Capital and Coast District Health Board, Wellington, Wellington, New Zealand

10.1136/jnnp-2019-anzan.47

Introduction Headache is a common problem in primary care and is one of the main reasons for general practitioners to 